\title{
Extending Semantic Long-Term Knowledge on the Basis of Episodic Short-Term Knowledge
}

\author{
Ian Voss (ivoss@techfak.uni-bielefeld.de) \\ Ipke Wachsmuth (ipke@techfak.uni-bielefeld.de) \\ Artificial Intelligence Group, Faculty of Technology, University of Bielefeld \\ D-33594 Bielefeld, Germany
}

\section{Introduction}

We describe a technical approach to the extension of knowledge representation in a semantic long-term memory (sLTM) by the use of conceptualised episodes of a shortterm memory. Herewith we established a specific strategy for the interplay between two memory systems. Our approach is motivated by recent findings in EEG observations on alpha and theta oscillations which reflect such an interplay between short-term and long-term memory systems (Sauseng et al., 2002; Klimesch, 1999).

The context of our work is a domain-specific task which is demonstrated in a virtual reality environment in cooperation with the animated agent Max (Kopp, Jung, Lessmann \& Wachsmuth). German natural language instructions are used to assemble an aeroplane with the wooden toy kit "Baufix".

\section{Method}

Based on a semantic net (SN) for knowledge representation in our sLTM, a sequence of user instructions is segmented into episodes reflecting aggregates coded in the SN. The representations of these episodes are made available in an episodic short-term memory (eSTM) and can be addressed for later use in an instruction like "and now do the same thing again". When giving an instruction like "and now do the same again but with a 5-hole bar", need arises for the modification of a conceptualized episode in the eSTM. With the use of semantic knowledge about the parts still available in the episode, this task has been realized recently (Voss, 2003).

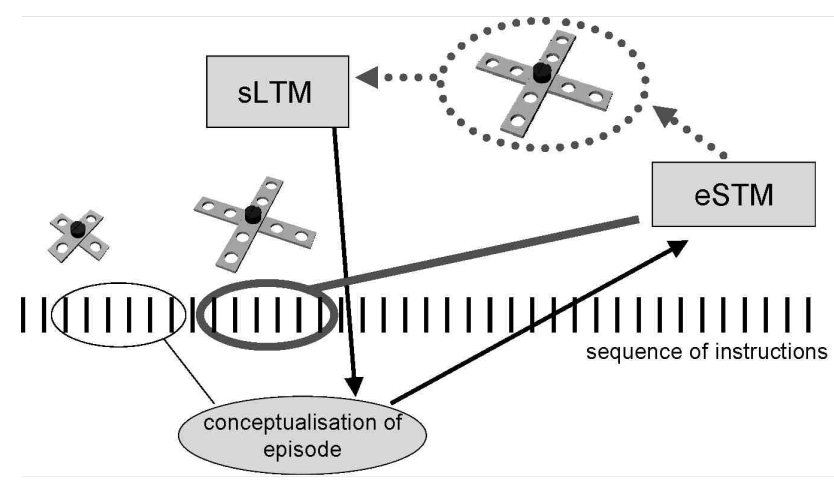

Figure 1: The original 3-hole-bar propeller is rebuilt as a 5hole-bar propeller and made available in the sLTM. Dotted lines indicate content and flow of information between a short-term and a long-term memory system.
As the modified episode has its roots in an episode that has been segmented on the basis of aggregate knowledge in the SN of the sLTM, the origin of the modified construction episode is available as a node in this $\mathrm{SN}$. We hence can augment the $\mathrm{SN}$ at the node that makes this new or modified aggregate available as an alternative one to its origin. Using the nomenclature of the COAR knowledge representation (Jung, 1998), we instantiate a new role-type (5-hole-bar propeller in Fig. 1) that is a specialisation of the same node as the original role-type (3-hole-bar propeller in Fig. 1).

The information flow for a propeller of a toy aeroplane and its modification is shown in Figure 1.

\section{Conclusion}

We established a method to augment the sLTM with unspecified information found in the user's instructions. This is realized by making use of structured representations in a semantic net on the one hand, and additional information that modifies a conceptualised episode with natural language instructions of a human instructor on the other hand.

\section{Acknowledgments}

This work is part of the Collaborative Research Centre SFB 360 at the University of Bielefeld and is partly supported by Deutsche Forschungsgemeinschaft (DFG). Implementation assistance by Marcel Richter is gratefully acknowledged.

\section{References}

Jung, B. (1998). Reasoning about Objects, Assemblies, and Roles in On-Going Assembly Tasks, Distributed Autonomous Robotic Systems 3, (pp. 257-266).

Klimesch, W. (1999). EEG alpha and theta oscillations reflect cognitive and memory performance: a review and analysis. Brain Res. Rev., 29 (pp. 169-195).

Kopp, S., Jung, B., Lessmann, N. \& Wachsmuth, I. Max A Multimodal Assistant in Virtual Reality Construction. KI-Themenheft "Embodies Conversational Agents", to be published.

Sauseng, P., Klimesch, W., Gruber, W., Doppelmayr, M., Stadler, W. \& Schabus, M. (2002). The interplay between theta and alpha oscillations in the human electroencephalogram reflects the transfer of information between memory systems, Neuroscience Letters, 324 (pp. 121-124).

Voss, I. (2003). Conceptualisation of Episodes in Interactive Virtual Construction. Human Computer Interaction, International, Crete; Adjunct Proceedings, in press. 